\title{
Maintenance of Mouse Spleen in Organ Culture and Assessment of Certain Functional Capabilities ${ }^{1}$
}

\author{
James T. Willerson, ${ }^{2}$ Jacquline R. Wakeland, \\ Marvin J. Stone, ${ }^{2}$ and Kern Wildenthal ${ }^{3}$ \\ Pauline and Adolph Weinberger Laboratory for Cardiopulmonary \\ Research and the Evelyn L. Overton Hematology-Oncology \\ Laboratory of the Department of Internal Medicine, \\ University of Texas Southwestern Medical \\ School at Dallas, Dallas, Texas 75235 , U.S.A.
}

Received December 18, 1973

To provide a means for studying certain immunological phenomena in mouse splenic tissue in vitro we have recently developed a system for maintaining mouse spleens in organ culture. Although previous attempts by some investigators to culture spleen tissue in organ culture have produced encouraging results (Globerson and Auerbach 1965, Globerson and Auerbach 1966, Holtermann and Nordin 1968, Klinman 1972, Pinkel 1963), others have reported difficulty in getting spleen organ cultures to survive in vitro, even when methods are used that enjoy considerable success with other types of tissue (Trowell 1959). The purpose of the present report is to describe the organ culture system we have found to be satisfactory and to define some of the properties of the cultured spleen fragments and whole spleens. Attention has been paid to possible reasons for the previously reported variability in the survival of splenic explants; in particular the influence of the size of the explant has been assessed and found to be of critical importance.

\section{Methods}

The general organ culture technique used by Dame Honor Fell and her associates at the Strangeways Research Laboratory (Fell 1964) and subsequently modified by Wildenthal (1971) for murine heart cultures was used as the prototype from which a murine spleen culture system could be developed. Adult male 6-week-old NIH GP mice were killed by cervical dislocation. The spleens were removed aseptically and transferred to a glass slide for viewing under a dissection microscope. Nonsplenic tissue was carefully trimmed away and blood was washed from the organ. The spleens were then explanted as the whole organs or sectioned horizontally into pieces of varying thicknesses $(0.5-2.0 \mathrm{~mm})$. The specimens were transferred to a shallow culture chamber containing $1.5 \mathrm{ml}$ of liquid medium.

The tissue was supported by a stainless-steel grid so that the bottom of the explant was exposed to the liquid and the top protruded into the atmosphere. The

1 This work was supported by grants from the American Heart Association and the NHLI (HL 14706).

2 Dr. Willerson is an Established Investigator of the American Heart Association.

2 Dr. Stone is an Established Investigator of the American Heart Association.

3 Dr. Wildenthal holds a USPHS Research Career Development Award (HL 70125). 
culture chambers were kept in Petri dishes placed in airtight McIntosh jars (Laboratory Thermal Equipment Ltd). The floors of the Petri dishes were covered with paper soaked with sterile distilled water to control humidity. The McIntosh jars were gassed with $95 \%$ oxygen and $5 \% \mathrm{CO}_{2}$ and incubated at $37.2 \pm 0.5^{\circ} \mathrm{C}$. The medium and atmosphere were renewed at 48 -hour intervals. The constituents of the medium included "medium 199", a prepared, chemically-defined media containing a balanced salt solution, glucose, certain amino acids, fats, vitamins, and other organic compounds (Grand Island Biological Co) (Morgan et al. 1950), fetal calf serum (Colorado Serum Co), highly-purified crystalline bovine insulin (Mann Research Laboratories), and penicillin $\mathrm{G}$ and streptomycin (Grand Island Biological Co). In additional experiments similar culture methods were used to culture intact spleens obtained from late-fetal mice (18-21 days' gestation).

The ability of the explants to metabolize glucose was assessed from measurements of the glucose concentration in the medium before and after each 48-hour culture period (glucose oxidase technique). The presence of mouse immunoglobulin in the media after cultivation of the spleens was assessed by using monospecific goat antimouse IgG1, IgG2, and IgM (Meloy Laboratories). Each antiserum gave only a single line versus normal mouse serum on immuno-electrophoretic analysis. Culture fluids were concentrated X25 in collodion bags (Schleicher-Schuell) under vacuum. Double diffusion-in-agar (Ouchterlony) analysis was carried out on $3 \times 2$ glass slides using $1.3 \%$ Noble agar (Difco Laboratories) and veronal buffer (ionic strength $0.05, \mathrm{pH} 8.6$ ). Plates were read at 6 hours and then at 24-hour intervals for 6 days before being discarded.

For histological studies, the splenic tissue was fixed in $10 \%$ formalin at varying intervals ( $2-8$ days) after explantation. The specimens were subsequently sectioned and stained with hematoxylin and eosin.

\section{Results}

Optimal medium: The initial culture medium chosen was "medium 199" supplemented with fetal calf serum and insulin $(50 \mathrm{mg} / \mathrm{ml})$. This medium proved satisfactory in preserving cell viability as judged by the histological appearance of the spleen fragments or whole spleens. Alterations in serum content between 10 and $50 \%$ were without apparent effect but elimination of all serum resulted in the earlier appearance and greater extent of histological necrosis. Cell viability as assessed histologically was also reduced by exclusion of insulin from the basic culture medium.

Neither addition of hydrocortisone $(0.1-10.0 \mathrm{mg} / \mathrm{ml})$ or triiodothyronine $(5 \times$ $\left.10^{-8}-5 \times 10^{-6} \mathrm{M}\right)$ to the medium, nor the elevation of glucose concentration from 100 to $500 \mathrm{mg} \%$ had any apparent effect. Similarly, variations in the oxygen content of the atmosphere between 20 and $95 \%$ resulted in no consistent changes in viability.

Thickness of spleen fragments: There was considerable variation in the viability of the explants depending on their size. As judged by gross and histological appearance, the larger spleen fragments (1.5-2.0 mm thickness) and whole spleens from adult mice survived better than did the smaller spleen fragments $(0.5-1.0 \mathrm{~mm}$ 


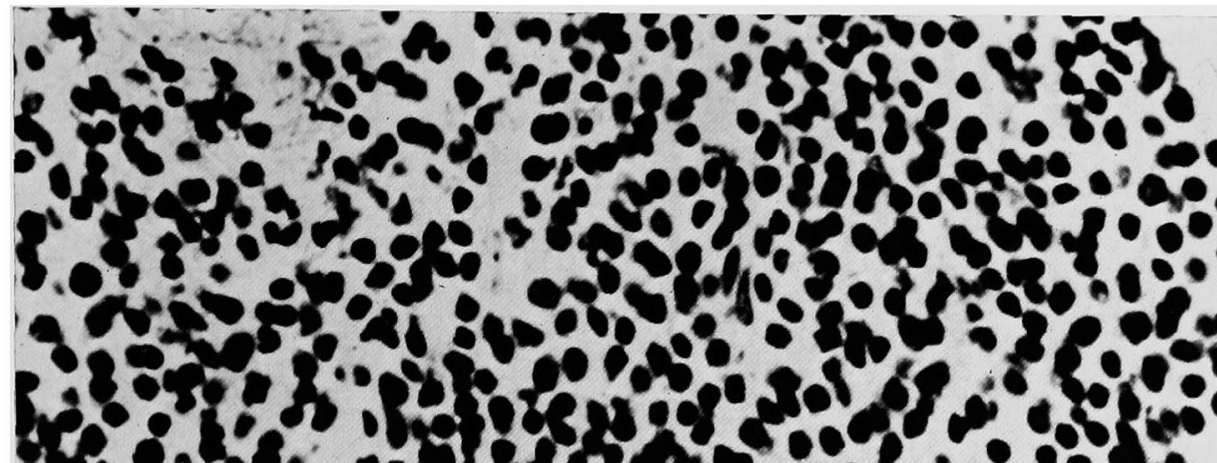

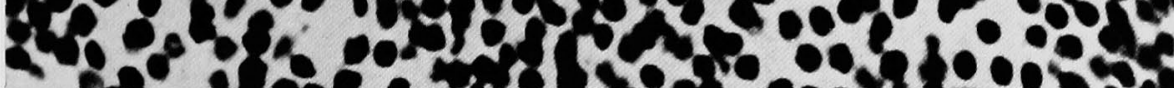

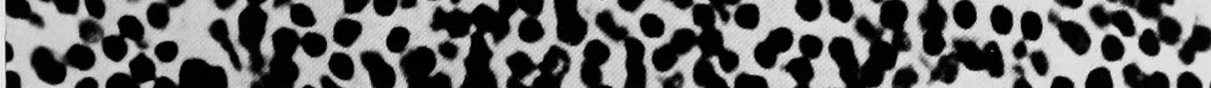

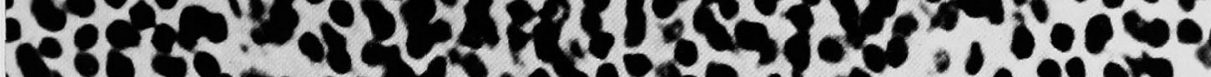

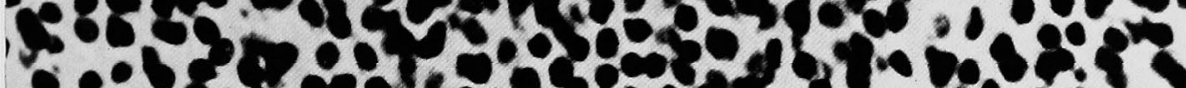

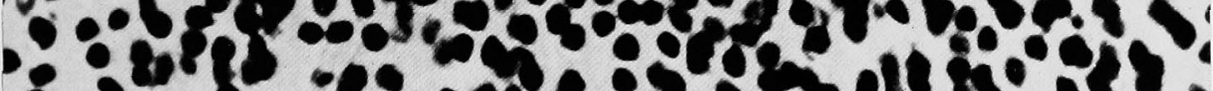

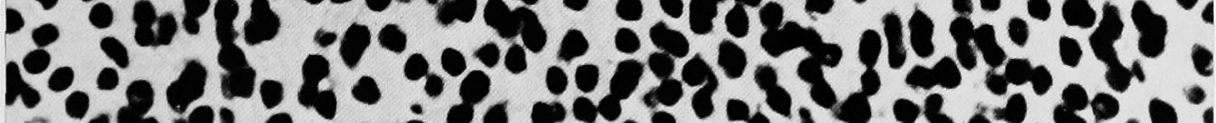

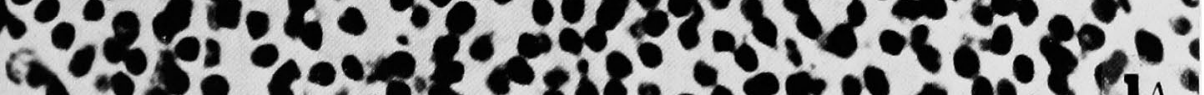

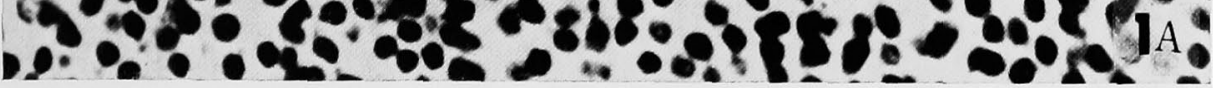

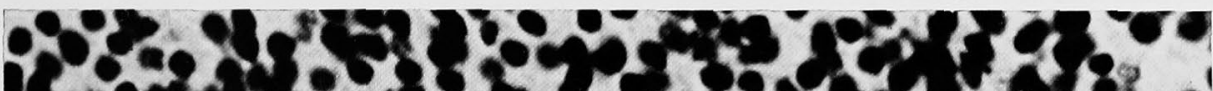

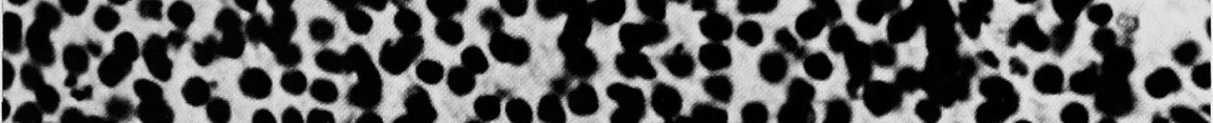

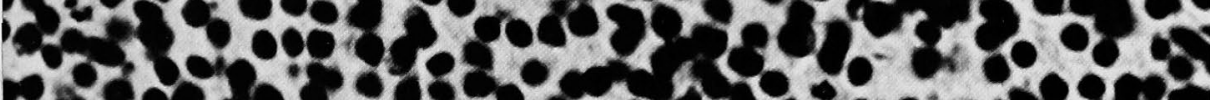

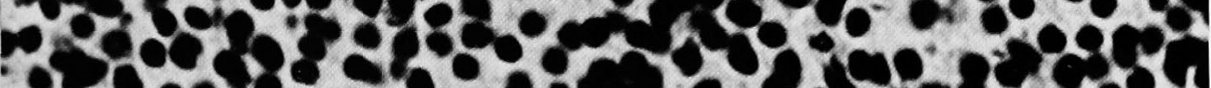

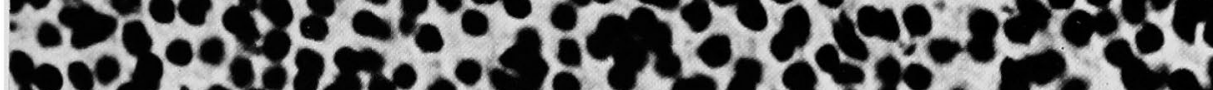

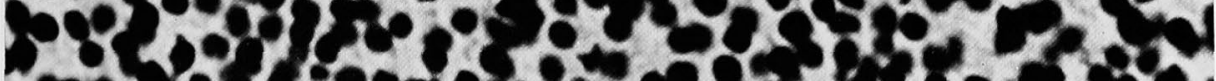

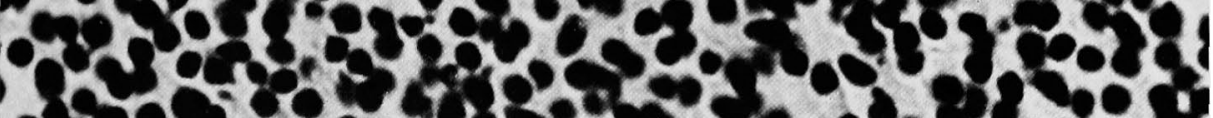
-3.

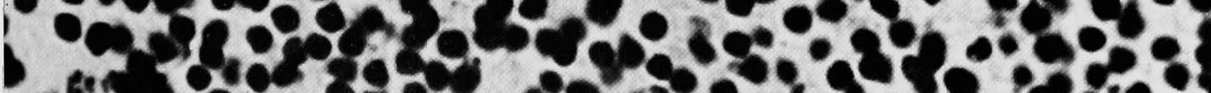

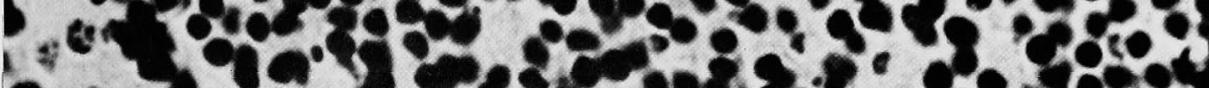

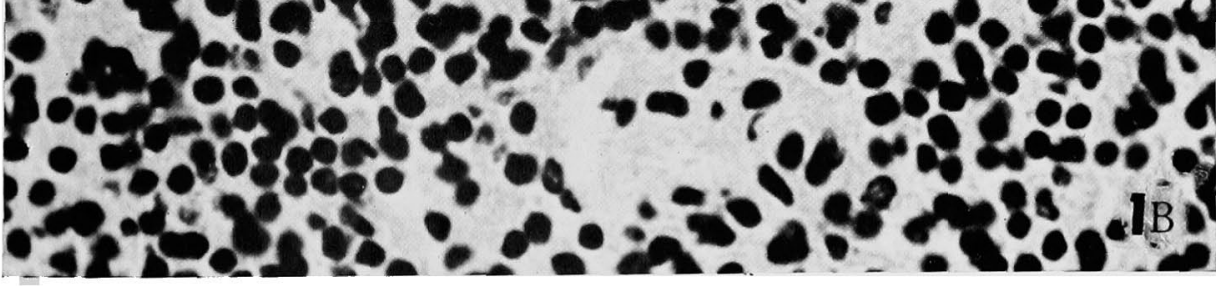

Fig. 1. Histological appearance of spleen fragments of $1.5 \mathrm{~mm}$ thickness maintained in organ culture for 2 days (panel A) and an adult whole spleen for 8 days (panel B). Note the predominance of small mononuclear cells and the large number of histologically viable cells. 


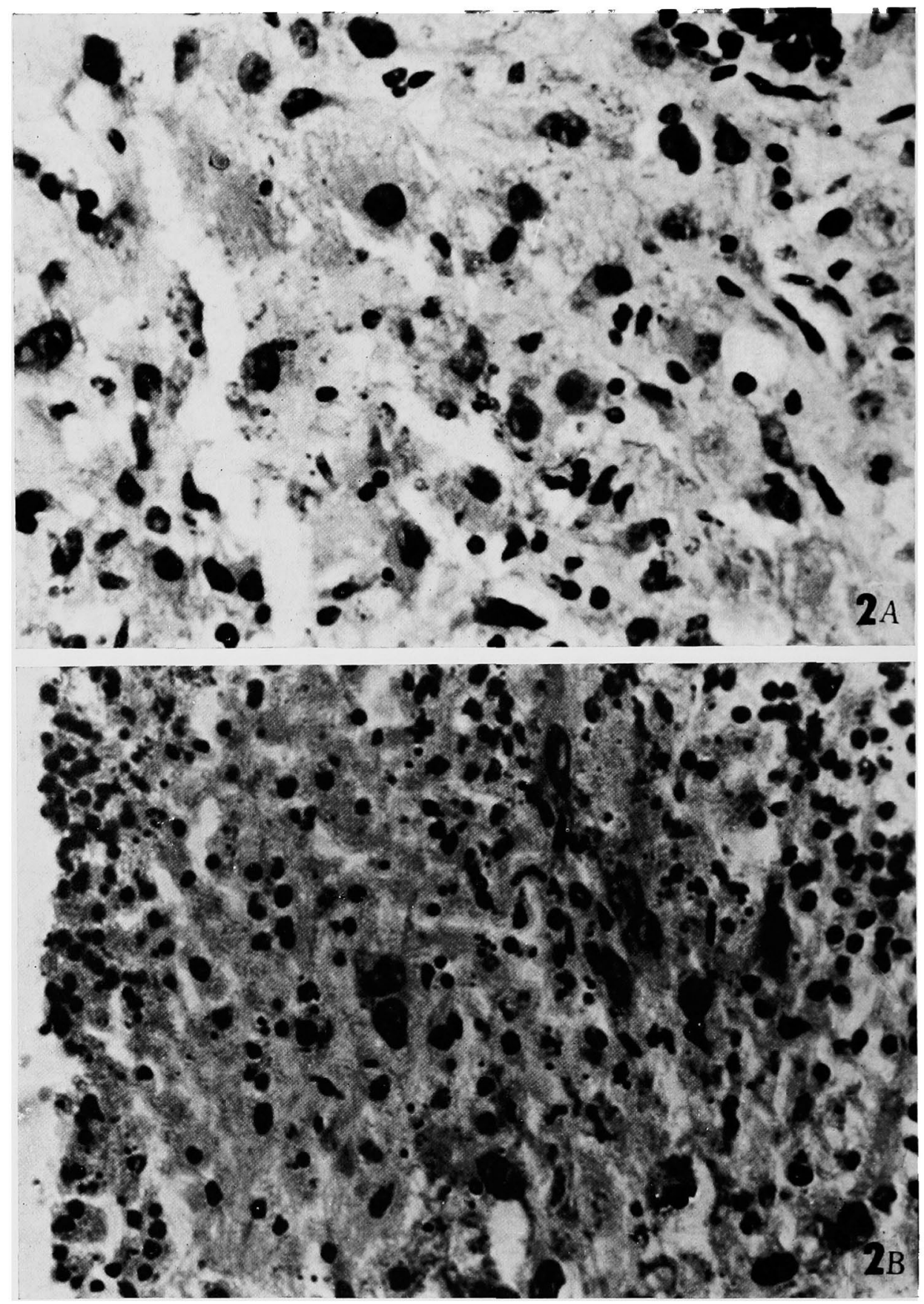

Fig. 2. Panel A. Intact spleen of fetal mouse $(0.4 \mathrm{~mm}$ thickness) following organ culture for 8 days. There are a relatively small number of histologically intact cells remaining. Panel B. Fragments of spleen of adult mouse $(0.5 \mathrm{~mm}$ thickness) following 6 days in organ culture. Few splenic cells persist in the culture specimen. 
thickness) for periods in excess of 4 days (Figs. 1, 2). Intact spleens of fetal mice (maximum thickness $0.4 \mathrm{~mm}$ ) displayed large numbers of normal-appearing lymphoid cells at the end of 2 days in culture but, like the smaller explants from adults, they did not survive well for longer periods. The horizontal dimensions of the cultured fragments (length and width) were unimportant in determining viability over a range of $2-10 \mathrm{~mm}$. The $1.5-2.0 \mathrm{~mm}$ spleen fragments and the whole adult spleens retained approximately $70 \%$ of their explantation weight following 4 days in organ culture and approximately $45-50 \%$ of their explantation weight after 8 days.

Gross and histological appearance of cultured spleen: Under optimal conditions the color of the spleen in culture remained the same as that of the organ in vivo for the first 2 days postexplantation. After 4 days most of the specimens had become pale and the predominant tint was light brown. This color persisted through the eighth day in most of the fragments. In those specimens that subsequent histological examinations confirmed as dead, the color had changed to opaque white and the tissue could be identified grossly as necrotic.

Histologically the viable splenic tissue that was cultured under optimal conditions was characterized by large numbers of apparently intact mononuclear cells for at least 8 days (Fig. 1). Occasional plasma cells and large numbers of reticulum cells were also present in these specimens. Between the second and eighth days in culture the number of lymphoid cells decreased. There were scattered areas of necrosis in the majority of the specimens but this was not extensive. The necrosis often involved one side of the specimen, ususally the underside, i.e., the one not exposed directly to the atmosphere. The amount of necrosis increased between the second and eighth days in organ culture, but even after 8 days greater than $80 \%$ of the explant appeared viable. Arrangement of the splenic architecture into "red pulp" and "white pulp" did not persist in vitro after 2 days.

Utilization of glucose by cultured spleen: Glucose was actively utilized by the $1.5 \mathrm{~mm}$ spleen fragments and whole adult spleens for the entire 8-day period of culture. There was only a small reduction in the total uptake of glucose between the second and eighth day, in keeping with the reduction in the over-all size and weight of the explant (Table 1). Glucose uptake was greater in $0.5 \mathrm{~mm}$ spleen fragments than in those of $1.5 \mathrm{~mm}$ thickness immediately following explantation but by 8 days glucose utilization in the fragments of $0.5 \mathrm{~mm}$ thickness had decreased much more than that of spleen fragments of greater thickness (Table 1). In a few experiments three spleen fragments of $0.5 \mathrm{~mm}$ thickness were stacked one upon another to provide a total thickness of $1.5 \mathrm{~mm}$. In the stacked spleens the reduction in glucose uptake with time resembled that noted for single spleen fragments of $1.5 \mathrm{~mm}$ thickness (Table 1).

Presence of immunoglobulins: Concentrated culture fluids were tested for reactivity against monospecific mouse antiimmunoglobulin sera (Table 2). After 2 days in culture, spleen fragments of either 0.5 or $1.5 \mathrm{~mm}$ thickness had released detectable quantities of immunoglobulin into the media (Table 2). By 6 days, however, media from $0.5 \mathrm{~mm}$ fragments had no detectable immunoglobulins, whereas $1.5 \mathrm{~mm}$ fragments had continued to release $\operatorname{IgG} 1, \operatorname{IgG} 2$, and $\operatorname{IgM}$. When three 
$0.5 \mathrm{~mm}$ fragments were stacked one on another to provide a total thickness of $1.5 \mathrm{~mm}$, the results resembled those for a single $1.5 \mathrm{~mm}$ fragment inasmuch as persistent, though reduced, antibody release was still present after 6 days in culture.

All of the precipitin lines in each of the above tests were evident by 16 hours in the Ouchterlony analyses. In no instance did the culture media react with any of the antisera.

Table 1. Glucose utilization by spleen fragments of different thicknesses

\begin{tabular}{|c|c|c|c|}
\hline $\begin{array}{l}\text { Specimens } \\
\text { (number of fragments }\end{array}$ & $\times$ thickness) & $\begin{array}{c}\text { Time } \\
\text { (days) }\end{array}$ & $\begin{array}{c}\text { Glucose } \\
\text { (nmoles } / \mathrm{hr} \pm 1 \mathrm{SD})\end{array}$ \\
\hline $1 \times 1.5 \mathrm{~mm}$ & & $0-2$ & $41 \pm 9.1$ \\
\hline $1 \times 1.5 \mathrm{~mm}$ & & $6-8$ & $26 \pm 4.5(63 \%)^{a}$ \\
\hline $1 \times 0.5 \mathrm{~mm}$ & & $0-2$ & $19 \pm 4.7$ \\
\hline $1 \times 0.5 \mathrm{~mm}$ & & $6-8$ & $7 \pm 5.3(37 \%)^{a}$ \\
\hline $3 \times 0.5 \mathrm{~mm}$ & $(\text { separate })^{b}$ & $0-2$ & $57 \pm 9.6$ \\
\hline $3 \times 0.5 \mathrm{~mm}$ & & $6-8$ & $22 \pm 9.2(39 \%)^{a}$ \\
\hline $3 \times 0.5 \mathrm{~mm}$ & $(\text { stacked })^{b}$ & $0-2$ & $31 \pm 4.5$ \\
\hline $3 \times 0.5 \mathrm{~mm}$ & & $6-8$ & $17 \pm 3.5(55 \%)^{a}$ \\
\hline
\end{tabular}

a Per cent of uptake during $0-2$ day interval.

${ }_{b}$ Three spleen slices of $0.5 \mathrm{~mm}$ thickness were explanted so that they lay on the culture grid without touching each other (separate) or were placed vertically on top of each other to mimic a single $1.5 \mathrm{~mm}$ slice (stacked).

Five separate specimens were cultured in each group, and media were collected at 2 days and at 8 days for glucose determinations.

Table 2. Immunoglobulin present in media after cultivation of spleen fragments of different thicknesses

\begin{tabular}{cccccc}
\hline \hline $\begin{array}{c}\text { Specimens } \\
\text { (number of fragments } \times \text { thickness) }\end{array}$ & $\begin{array}{c}\text { Time } \\
\text { (days) }\end{array}$ & IgG1 & IgG2 & IgM \\
\hline $1 \times 1.5 \mathrm{~mm}$ & $0-2$ & $2+$ & $1+$ & Trace \\
$1 \times 1.5 \mathrm{~mm}$ & $4-6$ & $1+$ & Trace & Trace \\
$1 \times 0.5 \mathrm{~mm}$ & & $0-2$ & $1+$ & Trace & None \\
$1 \times 0.5 \mathrm{~mm}$ & $4-6$ & None & None & None \\
$3 \times 0.5 \mathrm{~mm}$ & (separate) & $0-2$ & $2+$ & $1+$ & Trace \\
$3 \times 0.5 \mathrm{~mm}$ & & $4-6$ & None & None & None \\
$3 \times 0.5 \mathrm{~mm}$ & (stacked) & $0-2$ & $3+$ & $2+$ & Trace \\
$3 \times 0.5 \mathrm{~mm}$ & & 6 & Trace & Trace & None \\
\hline
\end{tabular}

See Table 1 for details of experimental protocol.

\section{Discussion}

These studies demonstrate that intact spleens from adult mice and splenic fragments of $1.5 \mathrm{~mm}$ thickness or greater can be maintained in organ culture for at least 8 days under appropriate conditions, and that the splenic cells utilize glucose and release immunoglobulins throughout that period of time. Focal areas of necrosis occur during the interval between 2 and 8 days in organ culture, and there is a decrease in the number of lymphoid cells. Nevertheless, large numbers of lymphoid cells survive and appear normal histologically even after 8 days in this organ culture model. 
The critical factor for insuring prolonged viability of the explants is a thickness of $1.5 \mathrm{~mm}$ or greater. This observation is at odds with the classical dictum of organ culture that survival in thick explants is reduced because of oxygen diffusion limitations, and thus that explants of $<1 \mathrm{~mm}$ thickness characteristically survive much better than larger ones (Moscona et al. 1965). The reason for enhanced survival of the larger spleen fragments is not clear. Perhaps some sort of massdependent "self conditioning" is especially important for splenic tissue in organ culture, as it is for some other tissues in cell culture systems (Gordon and Wilde 1965). It is also possible that the mild trauma involved in sectioning small spleen fragments results in premature death. On the other hand, whole spleens from fetal mice, which were also $<1.0 \mathrm{~mm}$ in diameter, behaved like the small fragments of adult spleens despite not being sectioned. Although different metabolic requirements of fetal tissues might be a factor in the failure of fetal spleens to thrive in culture, it also seems possible that their small size might be a major deterrent to prolonged survival in the present system. When 3 adult spleen fragments of $0.5 \mathrm{~mm}$ thickness were stacked one upon another their histological appearance, glucose uptake, and immunoglobulin release patterns tended to resemble those noted for a single fragment of $1.5 \mathrm{~mm}$ thickness, lending support to the probability that the total thickness of the explant per se is of critical importance for prolonged viability.

There are a few previous reports of organ cultures of mouse spleen fragments (Globerson and Auerbach 1965, Globerson and Auerbach 1966, Holtermann and Nordin 1968, Klinman 1972, Pinkel 1963). Globerson and Auerbach $(1965,1966)$ have described a "filter-well" system in which spleen fragments of $0.2-0.3 \mathrm{~mm}$ thickness survived histologically for 3-5 days. Primary antibody production against sheep red blood cells by some of the spleen fragments was demonstrated. Pinkel (1963) has reported successful long-term organ cultures using spleen fragments $2 \mathrm{~mm}$ in thickness obtained from weanling mice but no data on the functional capabilities of the explants were presented. Holtermann and Nordin (1968) described the ability of $1-1.5 \mathrm{~mm}$ murine spleen fragments to produce primary antibody to sheep red blood cells for several days in organ culture. Klinman (1972) has also used spleen fragments in organ culture to study the mechanism of antigenic stimulation of primary and secondary clonal precursor cells. Trowell (1959), on the other hand, reported that spleen organ cultures were singularly difficult to maintain in a viable state with his standard system in which pieces of unstated size were taken from spleens of newborn rats.

The present system provides a simple and effective means for maintaining spleen fragments of at least $1.5 \mathrm{~mm}$ thickness in vitro in a state in which histological appearance of the cells is nearly normal and in which functional activity is retained for relatively long periods.

\section{Summary}

A method for maintaining mouse spleen in organ culture has been described. Under appropriate conditions, the spleens survive for at least 8 days in vitro. The histological appearance of most of the cells remains normal, and glucose uptake and release of immunoglobulins persist during cultivation. Satisfactory results are 
dependent on utilization of relatively thick sections of splenic tissue: thin specimens $(<0.5 \mathrm{~mm}$ slices from adult spleen and whole spleens from fetal mice) fail to survive well, whereas slices of $>1.5 \mathrm{~mm}$ thickness or whole spleens from adult mice survive histologically and functionally for a period of at least 8 days.

\section{Acknowledgements}

The authors are indebted to Mrs. Susan Jasinski and Mrs. Phyllis Morton for performing the glucose determinations, to Mrs. Jennifer Fedak for helping with the immunoglobulin determinations, and to Miss Pat Wells for secretarial assistance.

\section{References}

Fell, H. B. 1964. The role of organ cultures in the study of vitamins and hormones. Vitamins and Hormones 22: 81-127.

Globerson, A., and Auerbach, R. 1965. Primary immune reactions in organ cultures. Science 149: 991-993.

— and -. 1966. Primary antibody response in organ cultures. J. Exp. Med. 124: 1001-1016.

Gordon, H. P., and Wilde, C. E., Jr. 1965. "Conditioned" medium and heart muscle differentiation: contrast between explants and disaggregated cells in chemically defined medium. Exp. Cell Res. 40: 438-442.

Holtermann, O. A., and Nordin, A. A. 1968. Primary induction of plaque forming antibody producing cells in spleen organ culture. Proc. Soc. Exp. Biol. Med. 127: 675-678.

Klinman, N. R. 1972. The mechanism of antigenic stimulation of primary and secondary clonal precursor cells. J. Exp. Med. 136: 241-260.

Morgan, J. F., Morton, H. J., and Parker, R. C. 1950. Nutrition of animal cells in tissue culture. I. Initial studies on a synthetic medium. Proc. Soc. Exp. Biol. Med. 73: 1-8.

Moscona, A., Trowell, O. A., and Willmer, E. N. 1965. Chapter 2. Methods. In "Cells and Tissues in Culture. Methods, Biology, and Physiology". Willmer, E. N., vol. I, p. 19. Academic Press, London and New York.

Pinkel, D. 1963. Successful cultivation of spleen fragments in organ culture. Proc. Soc. Exp. Biol. Med. 112: 242-247.

Trowell, O. A. 1959. The culture of mature organs in a synthetic medium. Exp. Cell Res. 16: $118-147$.

Wildenthal, K. 1971. Long-term maintenance of spontaneously beating mouse hearts in organ culture. J. Appl. Physiol. 30: 153-157. 